08.2

\title{
ПЭМ-исследование многослойных буферных структур AIN-AIGaN-GaN на кремниевых подложках
}

\author{
(c) А.В. Мясоедов, А.В. Сахаров, А.Е. Николаев, А.Е. Калмыков, Л.М. Сорокин, В.В. Лундин \\ Физико-технический институт им. А.Ф. Иофрфе РАН, Санкт-Петербург, Россия \\ E-mail: amyasoedov88@gmail.com
}

Поступило в Редакцию 8 июля 2020 г.

В окончательной редакции 8 июля 2020 г.

Принято к публикации 10 июля 2020 г.

\begin{abstract}
Методом просвечивающей электронной микроскопии исследованы две буферные структуры на основе растворов $\mathrm{Al}_{x} \mathrm{Ga}_{1-x} \mathrm{~N}$ : с легированием кремнием и без легирования. Структуры выращены на кремниевых подложках с ориентацией (111) методом газофазной эпитаксии из металлоорганических соединений с последовательно уменьшающимся содержанием Al. Установлено значительное снижение плотности прорастающих дислокаций в области промежуточных слоев при изменении доли Al с 32 до 23\%. Обнаружены фазовый распад и явление композиционной автомодуляции в слоях $\mathrm{Al}_{x} \mathrm{Ga}_{1-x} \mathrm{~N}$ в направлении роста при содержании $\mathrm{Al}$, равном 32, 23, 12 и 4\%. Предложена модель структуры слоев в области модуляции состава.
\end{abstract}

Ключевые слова: прорастающие дислокации, просвечивающая электронная микроскопия, газофазная эпитаксия из металлоорганических соединений, спонтанная модуляция состава.

DOI: 10.21883/PJTF.2020.19.50047.18457

Эпитаксиальные слои нитрида галлия $(\mathrm{GaN})$ широко используются в современной оптоэлектронике (светодиоды, лазеры) и СВЧ-микроэлектронике. При росте гетероструктур на основе нитрида галлия наибольшее распространение получили подложки сапфира и карбида кремния. С целью удешевления стоимости производства III-N гетероструктур в мире все чаще используют кремниевые подложки. Кроме низкой стоимости кремниевые подложки обладают более высокой по сравнению с сапфиром теплопроводностью, а также могут достигать в диаметре более $200 \mathrm{~mm}$.

Вследствие большой разницы коэффициентов теплового расширения кремниевой подложки и III-N слоев при остывании в эпитаксиальной структуре возникают растягивающие напряжения, что приводит к прогибу пластины и образованию трещин. Для предотвращения этих нежелательных эффектов в буферный слой гетероструктуры вводятся специальные переходные слои, создающие напряжения сжатия во время роста. В литературе приводятся различные наборы переходных слоев [1,2], однако базовым элементом таких буферных слоев являются слои $\mathrm{AlN}$ и $\mathrm{AlGaN}$. Из-за прямой реакции галлийсодержащих нитридов с подложкой кремния на интерфейсе всегда выращивается чистый $\mathrm{AlN}$, затем серия слоев $\mathrm{AlGaN}$ последовательно уменьшающегося состава. В зависимости от толщин и состава переходных слоев в финальной эпитаксиальной структуре можно получать как растягивающие, так и сжимающие напряжения.

Большое рассогласование постоянных решетки кремния и III-N приводит к очень высокой плотности дислокаций на интерфейсе и в буферном слое. При росте толстых слоев нитрида галлия плотность дислокаций уменьшается с набором толщины [3]. Кроме того, боль- шие напряжения сжатия могут дополнительно ускорять снижение плотности дислокаций. Для существенно более простой структуры $\mathrm{SiC}-\mathrm{AlGaN}-\mathrm{GaN}$ (стандартная последовательность буферных слоев при росте на $\mathrm{SiC}$ подложках) это относительно хорошо изученное явление [4], для более сложной многослойной буферной структуры на кремниевых подложках это явление требует более тщательного изучения.

Цель настоящей работы состоит в исследовании методом просвечивающей электронной микроскопии (ПЭМ) структурного состояния двух буферных систем, использованных при росте светоизлучающей и транзисторной приборных структур. Обе системы имели одинаковую последовательность буферных слоев $\mathrm{Al}_{x} \mathrm{Ga}_{1-x} \mathrm{~N}$, но разные верхние части. Для светоизлучающей структуры она состояла из активной области $\mathrm{InGaN} / \mathrm{GaN}$ и контактного слоя $p$-GaN, что типично для светодиодных приложений [5], для транзисторной структуры - из барьерного слоя $\mathrm{AlGaN}$, формирующего канал HЕМТ-структуры [6]. Однако данное различие не влияло на предмет исследования, поскольку приборные характеристики данных структур не являются предметом настоящей работы. Дополнительная разница состояла в том, что светоизлучающая структура легировалась кремнием, который сильно влияет на развитие напряжений в III-N эпитаксиальных слоях. Эпитаксиальные структуры выращивались методом МОС-гидридной эпитаксии (МОС - металлоорганические соединения) на установке АIX2000НT с планетарным реактором вместимостью шесть подложек диаметром 2 дюйма. Установка снабжена системой оптической in situ рефлектометрии. Аммиак, триметилгаллий, триэтилгаллий, триметилиндий, триметилалюминий, моносилан и биспентациклодиенил магния использовались в качестве источников. В качестве несу- 
Структурная модель исследуемых образцов

\begin{tabular}{c|c|c|c|c}
\hline $\mathrm{Al}_{x} \mathrm{Ga}_{1-x} \mathrm{~N}$ & $t, \mathrm{~nm}$ & $x$ & $d_{1}, \mathrm{~nm}$ & $d_{2}, \mathrm{~nm}$ \\
\hline $\mathrm{AlN}$ & 70 & 1.00 & - & - \\
$\mathrm{AlGaN} \mathrm{1}$ & 230 & 0.94 & - & - \\
$\mathrm{AlGaN} \mathrm{2}$ & 140 & 0.78 & - & - \\
$\mathrm{AlGaN} \mathrm{3}$ & 140 & 0.54 & - & - \\
$\mathrm{AlGaN} \mathrm{4}$ & 140 & 0.32 & $\sim 2.3$ & $\sim 4.5$ \\
$\mathrm{AlGaN} \mathrm{5}$ & 140 & 0.23 & $\sim 3.0$ & $\sim 6.0$ \\
$\mathrm{AlGaN} 6$ & 140 & 0.12 & $\sim 3.0$ & $\sim 6.0$ \\
$\mathrm{AlGaN} 7$ & 140 & 0.04 & $\sim 2.6$ & - \\
$\mathrm{GaN}$ & $500 / 900$ & 0.00 & - & -
\end{tabular}

Примечан ие. $t$ - толщины слоев (толщина слоя $\mathrm{GaN}$ составила $500 \mathrm{~nm}$ для случая светоизлучающей структуры и $900 \mathrm{~nm}$ для транзисторной), $x$ - содержание алюминия, $d_{1}$ и $d_{2}-$ периоды модуляции состава.

щих газов при росте $\mathrm{AlN} / \mathrm{AlGaN}$-буферных слоев и $n$ $\mathrm{GaN}$ применялся водород, при росте индийсодержащих слоев - азот, при росте $p$-AlGaN и $p$-GaN - азотводородная смесь.

Для обоих образцов на подложке $\mathrm{Si}(111)$ были выращены слой $\mathrm{AlN}(70 \mathrm{~nm})$, затем слой $\mathrm{AlGaN} 1$ (230 nm) с содержанием алюминия 94\% и шесть слоев $\mathrm{AlGaN}$ с последовательным уменьшением содержания алюминия до 4\% (слои $\mathrm{AlGaN} \mathrm{2-7).} \mathrm{Состав} \mathrm{в} \mathrm{слоях} \mathrm{AlGaN}$ изменялся соотношением потоков триметилгаллия и триметилалюминия [7]. Значения концентрации алюминия в составе слоев AlGaN 1-7 указаны в таблице [8].

Для проведения электронно-микроскопических исследований методом механической шлифовки с последующим утонением посредством ионного травления при ускоряющем напряжении $4 \mathrm{kV}$ были приготовлены поперечные срезы данных образцов. Исследование образцов проводилось с помощью электронного микроскопа Philips EM420 при ускоряющем напряжении $100 \mathrm{keV}$.

Оба образца имели гладкие границы на интерфейсе с кремниевой подложкой и высокую плотность дислокаций вблизи нее (рис. 1). Анализ электронограмм, полученных с области интерфейса, показал, что для образцов выполняются следующие эпитаксиальные соотношения: $\mathrm{Si}(111)\|\operatorname{AlGaN}(0001), \operatorname{Si}[1 \overline{1} 0]\| \operatorname{AlGaN}[11 \overline{2} 0]$.

Анализ ПЭМ-изображений поперечных сечений обеих буферных структур $\mathrm{AlGaN}$ при действующем отражении $\mathbf{g}=0002$ показал (рис. 1), что значительное снижение плотности прорастающих дислокаций (ПД) с ненулевой винтовой компонентой вектора Бюргерса $\mathbf{b}_{c}$ наблюдается в области интерфейса слоев $\mathrm{AlGaN} 4$ и $\mathrm{AlGaN} 5$ с концентрациями алюминия 32 и $23 \%$ соответственно. Снижение плотности ПД обусловлено отклонением линий ПД от направления роста, их сближением и реакцией между ними с образованием полупетель (рис. 1) или новой ПД с результирующим вектором Бюргерса, равным сумме векторов взаимодействующих дислокаций.

Отклонение ПД смешанного типа является результатом стремления энергии, связанной с ПД, к минимуму [9]. С одной стороны, эта энергия уменьшается при

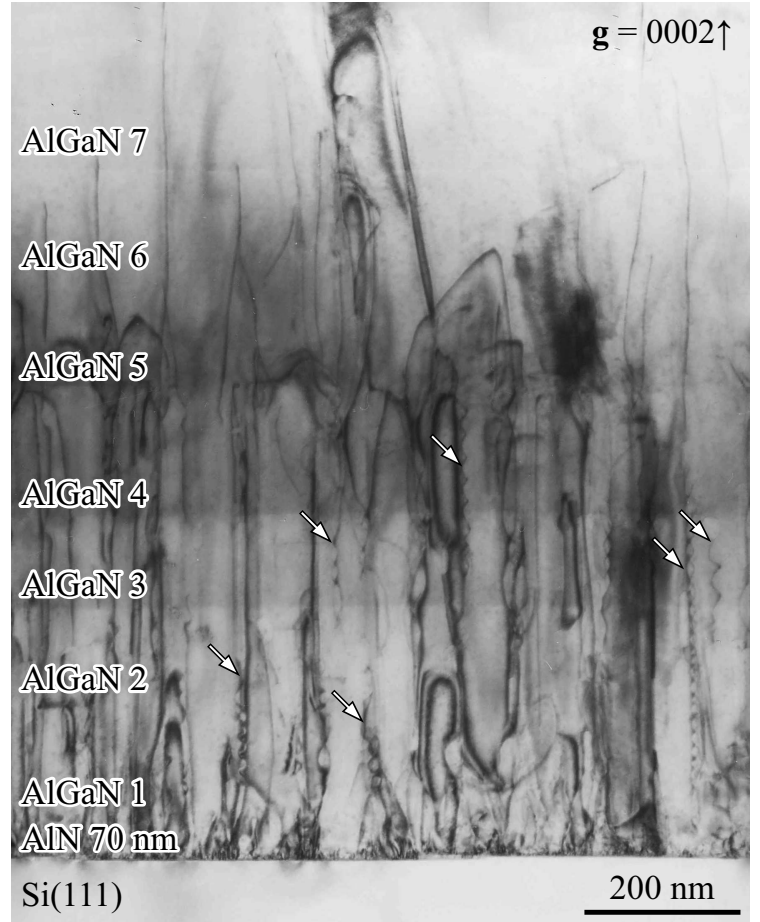

Рис. 1. ПЭМ-изображение буферной структуры $\mathrm{AlGaN}$ с последовательным уменьшением содержания $\mathrm{Al}$ при действующем отражении $\mathbf{g}=0002$. Стрелками отмечены геликоидальные дислокации.

увеличении винтовой составляющей ее вектора Бюргерca, т. е. линия дислокации стремится совпасть с вектором Бюргерса $\mathbf{b}_{a+c}=\mathbf{a}+\mathbf{c}$, где $\mathbf{a}$ и $\mathbf{c}-$ базисные векторы. C другой стороны, она уменьшается пропорционально сокращению длины дислокации, что достигается при совпадении линии дислокации с направлением роста слоя. В итоге направление линии ПД смешанного типа принимает промежуточное направление между вектором $\mathbf{a}+\mathbf{c}$ и направлением роста, совпадающим в данном случае с вектором с.

Кроме того, наклон линий ПД может быть связан с трехмерным режимом роста промежуточных слоев. В этом режиме ростовая поверхность образована пирамидальными фасетками. Если ПД оказывается вблизи такой фасетки, на нее начинают оказывать воздействие силы изображения [10], что приводит к отклонению линии ПД от направления роста гетероструктуры.

В слоях AlGaN 1-3 выявлены геликоидальные (спиральные) дислокации, которые полностью останавливаются на слое $\mathrm{AlGaN} 4$ (рис. 1). Они возникли, возможно, в результате взаимодействия винтовых дислокаций с точечными дефектами (вакансиями) [10]. В слое $\mathrm{AlGaN} 7$ выявлены отдельные частицы-преципитаты (см. вставку на рис. 2,a). Их появление обусловлено распадом твердого раствора в слое $\mathrm{AlGaN}$ - известная проблема, с которой сталкиваются при формировании структур AlGaN. Фазовый распад в тройных нитридных системах представляет собой сложное явление, на кото- 

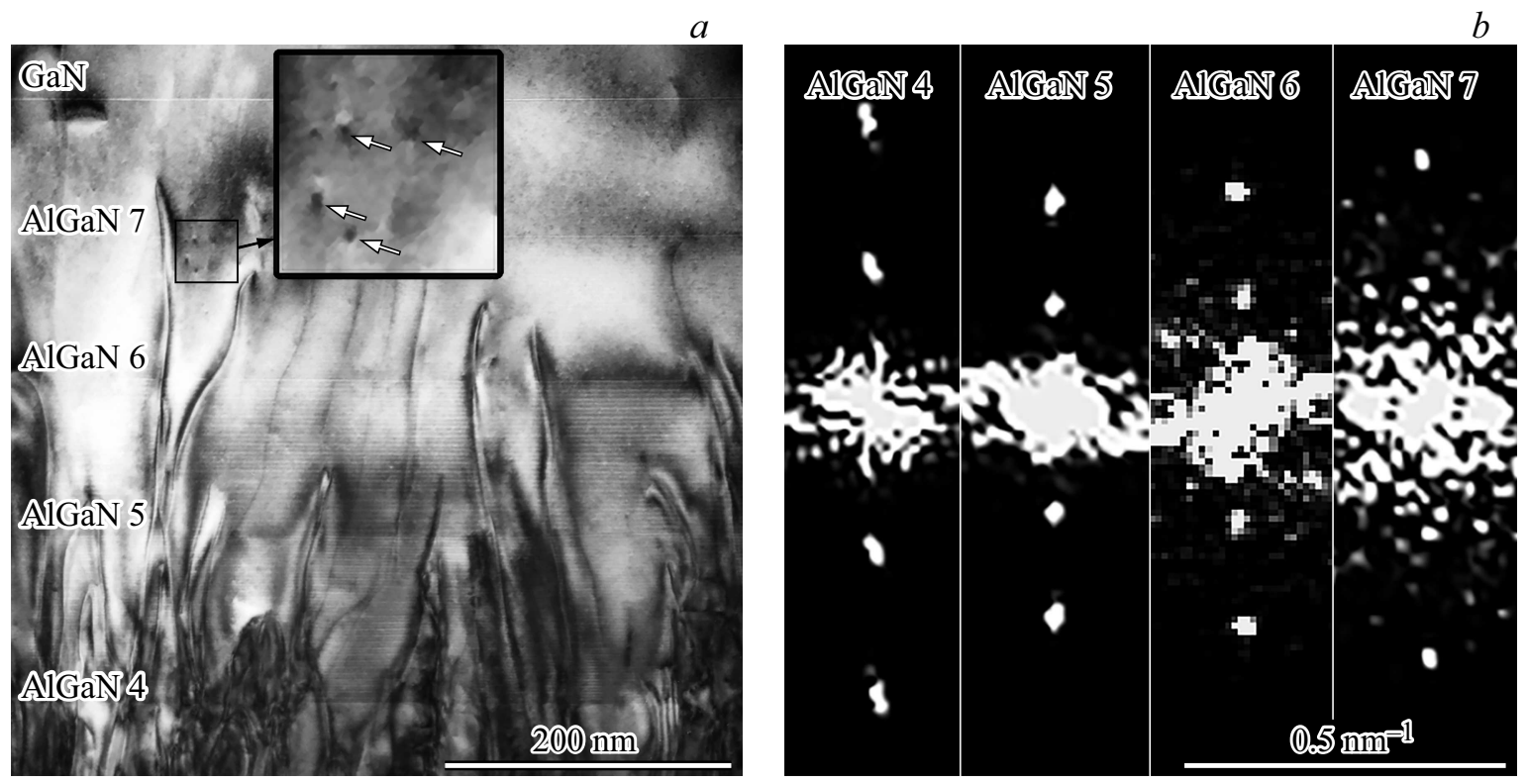

Рис. 2. $a-$ ПЭМ-изображение наблюдаемого упорядочения в слоях $\mathrm{AlGaN} \mathrm{4-7.} \mathrm{На} \mathrm{вставке} \mathrm{-} \mathrm{увеличенное} \mathrm{изображение} \mathrm{слоя}$ $\mathrm{AlGaN} 7$, стрелки указывают на частицы-преципитаты. $b-$ БПФ-изображения, полученные от областей, соответствующих слоям $\mathrm{AlGaN} 4-7$, приведенным на ПЭМ-изображении.

рое сильно влияют морфология ростовой поверхности и локальные напряжения [11].

В работе [12] отмечается, что эпитаксия соединений $\mathrm{A}_{3} \mathrm{~B}_{5}$ может сопровождаться неравновесным упорядочением двух типов (композиционной автомодуляцией и атомным порядком), которое связывается с реконструкцией поверхности. В частности, для соединений $\mathrm{AlGaN}$ встречается как композиционная автомодуляция [13], так и атомный порядок [14]. На рис. 2, $a$ приведено ПЭМ-изображение наблюдаемой периодической структуры в слоях $\mathrm{AlGaN} \mathrm{4-7,} \mathrm{обусловленной}$ композиционной автомодуляцией состава, аналогично работе [13]. Период модуляции определялся методом быстрого преобразования Фурье (БПФ). На рис. 2, $b$ приведены фрагменты БПФ-изображений, полученных от слоев $\mathrm{AlGaN} 4-7$. Видно, что положения пиков, соответствующих модуляции в слоях, заметно смещаются для слоев $\mathrm{AlGaN} 4$ и $\mathrm{AlGaN} 7$ относительно наблюдаемых для слоев $\mathrm{AlGaN} 5$ и $\mathrm{AlGaN}$ 6, для которых положение пиков существенно не меняется. Это смещение соответствует более короткому периоду модуляции в слоях AlGaN 4 и AlGaN 7.

Анализ профилей интенсивности дифракционного контраста участков изображения слоев, приведенных на ПЭМ-изображении (рис. 2, $a$ ), полученных вдоль направления роста буферной структуры, показал, что в слоях AlGaN 4-6 имеет место двойная модуляция, для которой можно определить $d_{1}$ как малый период и $d_{2}$ как большой период. На рис. 3, $a, b$ приведены примеры таких профилей для слоев $\mathrm{AlGaN} 5$ и $\mathrm{AlGaN} 4$ соответственно, из которых отчетливо видно чередование малого и большого максимумов интенсивности. Наблю- даемый практически линейный общий спад интенсивности обусловлен увеличением толщины просвечиваемой области вдоль линии построения профиля. На профиле интенсивности, приведенном на рис. $3, a$, мы присваиваем большим и малым максимумам составы $\mathrm{Al}_{x} \mathrm{Ga}_{1-x} \mathrm{~N}$ и $\mathrm{Al}_{z} \mathrm{Ga}_{1-z} \mathrm{~N}$ соответственно, а минимумам - состав $\mathrm{Al}_{y} \mathrm{Ga}_{1-y} \mathrm{~N}$; значения $x, y$ и $z$ отличаются друг от друга, что иллюстрируется схематической моделью на рис. $3, c$ (пропорции не соблюдены). Таким образом, малый период $d_{1}$ соответствует расстоянию между большим и малым максимумами, а большой период $d_{2}$ - расстоянию между двумя большими максимумами. Согласно нашим результатам, большой и малый периоды соотносятся как 1 к 2 , т.е. $d_{2}=2 d_{1}$. В таблице приведены значения периодов $d_{1}$ и $d_{2}$, полученные методом БПФ, для модуляции состава в слоях $\mathrm{AlGaN} 4-7$. Для слоя $\mathrm{AlGaN} 7$ наличие большого периода точно не установлено. Корреляцию между составом промежуточного слоя и величиной периода модуляции выявить не удалось. Если при снижении $x$ от 0.32 до 0.23 малый период увеличивается от $\sim 2.3$ до $\sim 3 \mathrm{~nm}$ (см. таблицу), то при дальнейшем снижении содержания $\mathrm{Al}$ период остается неизменным, а затем даже уменьшается. Отсутствие корреляции, по-видимому, связано с тем, что параметры сверхрешетки зависят не столько от состава, сколько от скорости роста [13].

На рис. 3, $d$ приведена электронограмма, полученная с области модуляции состава в слоях AlGaN 4-7 вдоль оси зоны $[1 \overline{1} 00]$, на которой наблюдается запрещенное отражение 0001. Аналогичное возбуждение данного отражения отмечалось в работах [13-15] и объяснялось чередованием слоев с различным содержанием Al. 


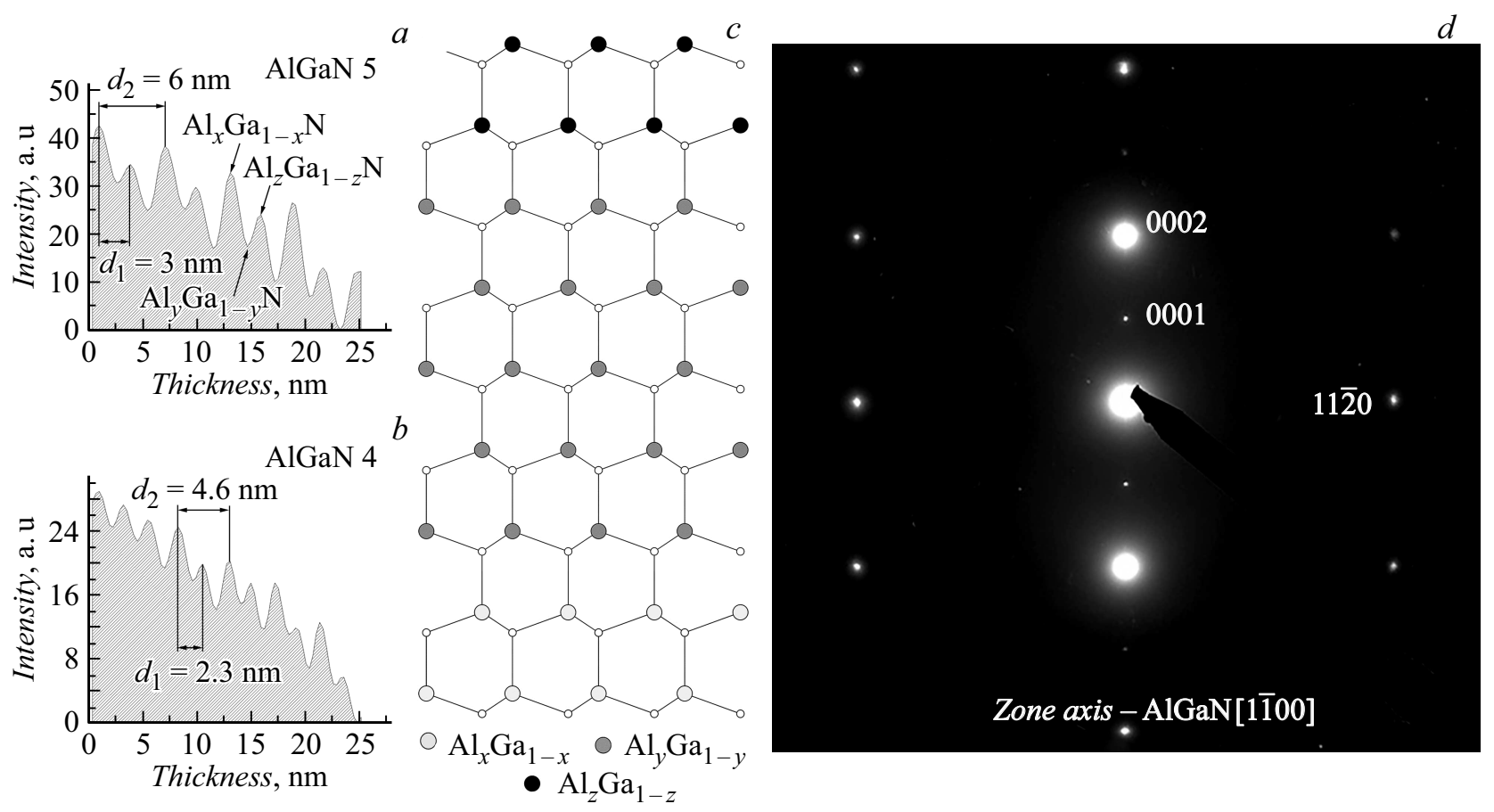

Рис. 3. $a$ и $b$ - примеры профилей интенсивности, полученные соответственно от областей, отвечающих слоям AlGaN 5 и $\mathrm{AlGaN} 4$, приведенным на ПЭМ-изображении (рис. 1); $c$ - схематическая модель упорядочения; $d-$ электронограмма, соответствующая области модуляции состава (слои AlGaN 4-7).

На рис. 3, представлена модель, которая объясняет это явление: запрещенный рефлекс вызван отражениями от рядом лежащих атомных слоев с различным составом $\mathrm{Al}_{x} \mathrm{Ga}_{1-x} \mathrm{~N}$.

Таким образом, анализ данных, полученных методом ПЭМ, показал, что использование буферных структур $\mathrm{Al}_{x} \mathrm{Ga}_{1-x} \mathrm{~N}$ на кремниевых подложках с последовательным уменьшением содержания Al при определенных условиях может вызывать отклонение ПД от направления роста с последующим их взаимодействием.

В слоях $\mathrm{Al}_{x} \mathrm{Ga}_{1-x} \mathrm{~N}$ с содержанием алюминия в диапазоне $0.04<x<0.32$ обнаружена спонтанная модуляция состава в слоях с двойным периодом, предложена модель строения образовавшейся в результате модуляции сверхрешетки.

\section{Благодарности}

Исследования методом ПЭМ выполнены с использованием оборудования федерального ЦКП „Материаловедение и диагностика в передовых технологиях“, поддержанного Минобрнауки России.

\section{Конфликт интересов}

Авторы заявляют, что у них нет конфликта интересов.

\section{Список литературы}

[1] Su J., Armour E., Lee S.M., Arif R., Papasouliotis G.D. // Phys. Status Solidi A. 2016. V. 213. N 4. P. 856-860. DOI: $10.1002 /$ pssa.201532708

[2] Rudinsky M.E., Lobanova A.V., Karpov S.Y., Talalaev R.A. // Jpn. J. Appl. Phys. 2019. V. 58. N SC. P. SC1017. DOI: $10.7567 / 1347-4065 / A B 06 B 7$

[3] Albrecht M., Nikitina I.P., Nikolaev A.E., Melnik Y.V., Dmitriev V.A., Strunk H.P. // Phys. Status Solidi A. 1999. V. 176. N 1. P. 453-458. DOI: $10.1002 /($ SICI) $1521-$ 396X(199911) 176:1<453::AID-PSSA453>3.0.CO;2-M

[4] Rudinsky M.E., Yakovlev E.V., Lundin W.V., Sakharov A.V., Zavarin E.E., Tsatsulnikov A.F., Velikovskiy L.E. // Phys. Status Solidi A. 2016. V. 213. N 10. P. 2759-2763. DOI: $10.1002 /$ pssa.201600210

[5] Сахаров А.В., Лундин В.В., Заварин Е.Е., Синицын М.А., Николаев А.Е., Усов С.О., Сизов В.С., Михайловский Г.А., Черкашин Н.А., Нуtсh М., Ние F., Яковлев Е.В., Лобанова А.В., Цачульников А.Ф. // ФТП. 2009. Т. 43. В. 6. C. 841-846.

[6] Цаиульников А.Ф., Сахаров А.В., Лундин В.В., Николаев А.Е., Заварин Е.Е., Синицын М.А., Яговкина М.А., Устинов В.М. // Мокеровские чтения. Науч.-практ. конф. по физике и технологии наногетероструктурной СВЧэлектроники. М.: НИЯУ МИФИ, 2011. С. 18.

[7] Лундин В.В., Николаев А.Е., Сахаров А.В., Брунков П.Н., Заварин Е.Е., Цаиульников А.Ф. // Письма в ЖТФ. 2010. Т. 36. В. 24. С. 33-39. [Пер. версия: $10.1134 / \mathrm{S} 1063785010120205]$. 
[8] Verkhovtceva E.V., Nikolaev A.E., Sakharov A.V., Sokolov R.V., Yagovkina M.A. // 11th Biennial Conf. on high resolution $X$-ray diffraction and imaging (XTOP 2012). St. Petersburg, 2012. P. 251-252.

[9] Mathis S.K., Romanov A.E., Chen L.F., Beltz G.E., Pompe W., Speck J.S. // J. Cryst. Growth. 2001. V. 231. N 3. P. 371-390. DOI: $10.1016 / \mathrm{S} 0022-0248(01) 01468-3$

[10] Hull D., Bacon D.J. // Introduction to dislocations. Butterworth-Heinemann, 2011. $272 \mathrm{p}$.

[11] Mayboroda I.O., Knizhnik A.A., Grishchenko Yu.V., Ezubchenko I.S., Zanaveskin M.L., Kondratev O.A., Presniakov M.Yu., Potapkin B.V., Ilyin V.A. // J. Appl. Phys. 2017. V. 122. N 10. P. 105305. DOI: $10.1063 / 1.5002070$

[12] Максимов К.С., Максимов С.К. // Письма в ЖТФ. 1998. T. 24. B. 10. C. $70-75$.

[13] Gao M., Bradley S.T., Cao Y., Jena D., Lin Y., Ringel S.A., Hwang J., Schaff W.J., Brillson L.J. // J. Appl. Phys. 2006. V. 100. N 10. P. 103512 . DOI: $10.1063 / 1.2382622$

[14] Cheng S., Langelier B., Ra Y.H., Rashid R.T., Mi Z., Botton G.A. // Nanoscale. 2019. V. 11. N 18. P. 8994-8999. DOI: $10.1039 / \mathrm{c} 9 \mathrm{nr} 01262 \mathrm{a}$

[15] Albrecht M., Lymperakis L., Neugebauer J., Northrup J.E., Kirste L., Leroux M., Grzegory I., Porowski S., Strunk H.P. // Phys. Rev. B. 2005. V. 71. N 3. P. 035314. DOI: 10.1103/PhysRevB.71.035314 